\title{
The influence of positive end-expiratory pressure on stroke volume variation in patients undergoing cardiac surgery: An observational study
}

\author{
Woon-Seok Kang, MD, ${ }^{\text {a,b }}$ Seong-Hyop Kim, MD, PhD, ${ }^{\text {a,b }}$ Sung Yun Kim, MD, ${ }^{a}$ Chung-Sik Oh, MD, ${ }^{a}$ \\ Song-Am Lee, MD, ${ }^{\text {b,c }}$ and Jun-Seok Kim, MD, $\mathrm{PhD}^{\mathrm{b}, \mathrm{c}}$
}

Objectives: Measurements of stroke volume variation for volume management in mechanically ventilated patients are influenced by various factors, such as tidal volume, respiratory rate, and chest/lung compliance. However, research regarding the effect of positive end-expiratory pressure on stroke volume variation is limited.

\begin{abstract}
Methods: Patients were divided into responder and nonresponder groups according to the prediction of fluid response by the passive leg raising test and hemodynamic parameters, including stroke volume variation, measured in all patients at the following ventilator settings: (1) conventional ventilation $(\mathrm{C})$, tidal volume $10 \mathrm{~mL} \cdot \mathrm{kg}^{-1}$ with positive end-expiratory pressure settings of $0(\mathrm{C} 0), 5(\mathrm{C} 5)$, and $10 \mathrm{cmH}_{2} \mathrm{O}(\mathrm{C} 10)$ and (2) lung protective ventilation $(\mathrm{P})$, tidal volume $6 \mathrm{~mL} \cdot \mathrm{kg}^{-1}$ with positive end-expiratory pressure settings of 0 (P0), 5 (P5), and $10 \mathrm{cmH}_{2} \mathrm{O}(\mathrm{P} 10)$.
\end{abstract}

Results: Regardless of ventilator setting, stroke volume variation in the responder group had an increasing trend as increased positive end-expiratory pressure level and was significantly higher than in the nonresponder group at each positive end-expiratory pressure level. The area under the curve was (1) 0.899 at C0, 0.942 at C5, and 0.985 at $\mathrm{C} 10$; and (2) 0.901 at $\mathrm{P} 0,0.932$ at P5, and 0.947 at P10. Optimal threshold values given by receiver operating characteristic curve analysis were (1) $13.5 \%, 13.5 \%$, and $14.5 \%$; and (2) $13.5 \%, 13.5 \%$, and $14.5 \%$, respectively.

Conclusions: The threshold value of stroke volume variation in predicting fluid responsiveness may change when positive end-expiratory pressure $10 \mathrm{cmH}_{2} \mathrm{O}$ is applied. This must be considered when stroke volume variation is used to detect the fluid responsiveness to prevent volume overload in this mechanical ventilation setting. (J Thorac Cardiovasc Surg 2014;148:3139-45)

See related commentary on pages 3146-7.

Fluid management is an essential component for the successful treatment of patients in the intensive care unit (ICU); therefore, the precise assessment of the patient's actual volume status and fluid responsiveness is important. The parameters for volume status and fluid responsiveness are usually the basis for deciding on the best initial treatment (ie, fluid administration vs medication) for hemodynamic

\footnotetext{
From the Department of Anesthesiology and Pain Medicine, ${ }^{a}$ Konkuk University Medical Center, Konkuk University School of Medicine, Seoul, Korea; Institute of Biomedical Science and Technology, ${ }^{\mathrm{b}}$ Konkuk University School of Medicine, Seoul, Korea; and Department of Thoracic and Cardiovascular Surgery, ${ }^{\mathrm{c}}$ Konkuk University Medical Center, Konkuk University School of Medicine, Seoul, Korea. This work was supported by Konkuk University.

Disclosures: Authors have nothing to disclose with regard to commercial support. Received for publication April 17, 2014; revisions received June 11, 2014; accepted for publication July 19, 2014; available ahead of print Sept 12, 2014.

Address for reprints: Seong-Hyop Kim, MD, PhD, Department of Anesthesiology and Pain Medicine, Konkuk University Medical Center, Konkuk University Hospital, 120-1, Neungdong-ro, Hwayang-dong, Gwangjin-gu, Seoul 143-729, Korea (E-mail: yshkim75@daum.net).

$0022-5223 / \$ 36.00$

Copyright (C) 2014 by The American Association for Thoracic Surgery

http://dx.doi.org/10.1016/j.jtcvs.2014.07.103
}

instability. In particular, in a patient undergoing cardiac surgery, fluid management is critical and complex because of the underlying cardiac pathology, concurrent medications, and factors that can influence cardiopulmonary bypass, such as inflammation or hormonal response. ${ }^{1}$

Continuous measurements of hemodynamic parameters, such as pulse pressure variation (PPV) and stroke volume variation (SVV), as indicators for fluid management have shown excellent results when compared with the traditional parameters, such as measurement of central venous pressure (CVP) and pulmonary capillary artery wedge pressure (PCWP) ${ }^{2,3}$ In mechanically ventilated patients, as with CVP and PCWP, PPV and SVV are influenced by tidal volume (TV), respiratory rate (RR), chest/lung compliance, and other factors, although to a lesser extent. ${ }^{4-6}$ To date, few studies have examined the effect of positive end-expiratory pressure (PEEP) on measurements of PPV and SVV.

The aim of the present study was to evaluate the effect of different ventilator settings and PEEP levels on the parameters used in monitoring volume status in patients undergoing cardiac surgery with mechanical ventilator support.

We hypothesized that different TV and PEEP levels in the same patient might alter absolute SVV values, and we 


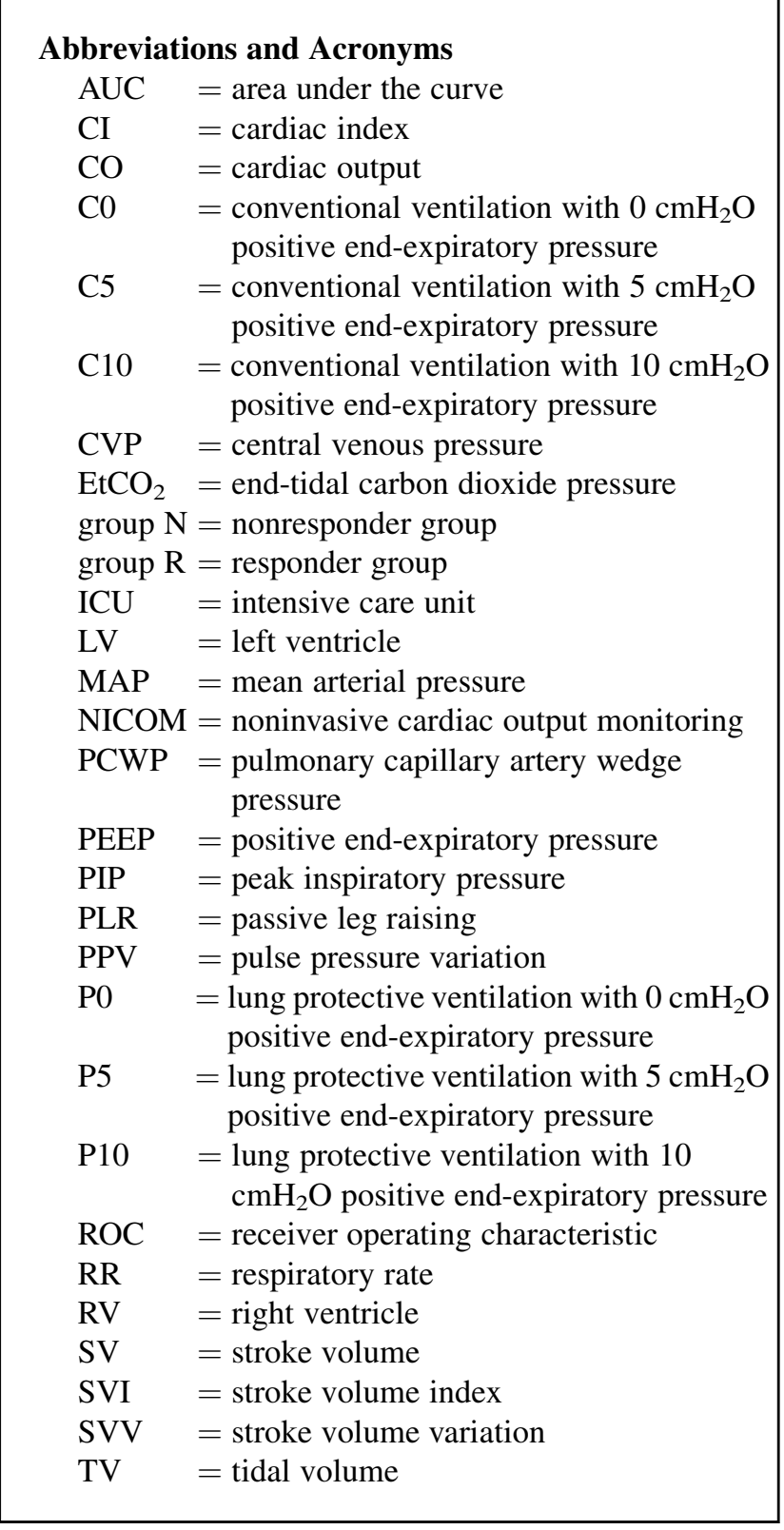

compared and analyzed the SVV values measured by bioreactance technique using a noninvasive cardiac output monitoring (NICOM) instrument at each TV and PEEP level.

\section{MATERIALS AND METHODS \\ Study Population}

Institutional review board approval (KUH1160028, September 2011) was obtained, the trial was registered at http://cris.nih.go.kr (KCT0000251), and all patients gave written, informed consent.

Patients undergoing aortic valve repair surgery for aortic valve stenosis or aortic valve insufficiency were enrolled prospectively from September 2011 to August 2013. Exclusion criteria was as follows: (1) urgent or emergency cases; (2) when combined with other concurrent valvular surgery; (3) patient age less than 16 years; (4) reduced left and right ventricular function (ejection fraction $<40 \%$ ); (5) postoperative dysrhythmia; (6) previous respiratory disease; (7) severe renal disease; (8) severe hepatic disease; or (9) dermatologic disease preventing application of patches for NICOM.

\section{Noninvasive Cardiac Output Monitoring in the Intensive Care Unit}

All patients were transferred to the ICU after surgical procedures. Patients were fully sedated with remifentanil and mechanically ventilated in a volume-control mode with standard settings to achieve normocapnea indicated by end-tidal carbon dioxide pressure $\left(\mathrm{EtCO}_{2}\right) 35$ to $45 \mathrm{~mm} \mathrm{Hg}$ by capnography (IntelliVue MP50; Philips Medizin Systeme, Boeblingen, Germany) according to the standard institutional ICU protocols. Before the present study, there were no additional interventions (eg, adjustments of fluid administration or medications). Invasive systemic arterial blood pressure, pulmonary arterial blood pressure, and CVP were continuously monitored in the ICU. After maintenance of mean arterial pressure (MAP) greater than $60 \mathrm{~mm} \mathrm{Hg}$, which is measured by invasive monitoring, and cardiac index $(\mathrm{CI})$ greater than $2.0 \mathrm{~L} \cdot \mathrm{min}^{-1} \cdot \mathrm{m}^{-2}$ for 1 hour, which is measured by pulmonary artery catheter (Swan-Ganz CCOmbo $\mathrm{CCO}$ / $\mathrm{SvO}$; Edwards Lifesciences, Irvine, Calif), the NICOM electrodes were placed on the patient's chest and connected to the NICOM controller (NICOM; Cheetah Medical Inc, Vancouver, Wash). The proximal electrodes were placed at the mid-subclavian region, and the distal electrodes were placed at the mid-portion of the lower costal margin. After initial calibration of the NICOM system, cardiac output (CO), CI, stroke volume (SV), stroke volume index (SVI), and SVV were monitored continuously.

The NICOM system's signal processing unit determines the relative phase shift $(\Phi)$ between input and output signals. The phase shift between input and output signals is due to changes in blood volume in the aorta. The SV determined by the NICOM can be estimated by $\mathrm{SV}=\mathrm{C} \cdot \mathrm{VET} \cdot \mathrm{d} \Phi / \mathrm{dt}_{\max }$, where $\mathrm{C}$ is a constant of proportionality, VET is ventricular ejection time, and $\mathrm{d} \Phi / \mathrm{dt}_{\max }$ is the peak rate of change of $\Phi{ }^{7}$ The value of $\mathrm{C}$ has been optimized in prior studies and accounts for patient age, gender, and body size. ${ }^{8}$ Maximal and minimal values of SV are determined beat-to-beat over a single respiratory cycle. The SVV is calculated as follows:

$$
\operatorname{SVV}(\%)=(\operatorname{SVmax}-\mathrm{SVmin}) /(\mathrm{SVmax}+\mathrm{SVmin} / 2) 100 .
$$

The value of SVV displayed by the device was the mean value for 1 minute, and the time interval between each measurement was 1 minute.

\section{Study Protocols}

The trial design of the study was parallel, and the patients were allocated (allocation ratio $=1: 1)$ to the responder group (group $\mathrm{R}$ ) or nonresponder group (group $\mathrm{N}$ ). To define a patient as a responder or a nonresponder, the passive leg raising (PLR) test was performed in the ICU by an assistant who lifted the patient's lower limbs in a straight manner to $45^{\circ}$ for 5 minutes. If the $\mathrm{CO}$ measured by NICOM was increased to more than $7 \%$ from the baseline value, the patient was included in group R., ${ }^{9,10}$

Regardless of the group the patient was in, 2 different mechanical ventilation strategies with escalating PEEP values were applied as follows: (1) conventional ventilation (C) with $\mathrm{TV}$ of $10 \mathrm{~mL} \cdot \mathrm{kg}^{-1}$ according to ideal body weight [50 (female: 45.5$)+0.91 \cdot($ height -152.4$)]$ and an RR to maintain an $\mathrm{EtCO}_{2}$ of 35 to $45 \mathrm{~mm} \mathrm{Hg}$ using capnography and PEEP settings of $0(\mathrm{C} 0), 5(\mathrm{C} 5)$, and $10 \mathrm{cmH}_{2} \mathrm{O}(\mathrm{C} 10)$; and (2) lung protective ventilation $^{11}(\mathrm{P})$ with $\mathrm{TV}$ of $6 \mathrm{~mL} \cdot \mathrm{kg}^{-1}$ according to ideal body weight, adequate $\mathrm{RR}$ to maintain same level of $\mathrm{EtCO}_{2}$ as conventional ventilation, and PEEP settings of 0 (P0), 5 (P5), and $10 \mathrm{cmH}_{2} \mathrm{O}$ (P10). The hemodynamic parameters measured were (1) MAP, heart rate, CVP, mean pulmonary arterial pressure, and PCWP derived by invasive arterial pressure monitoring device and pulmonary artery catheter; (2) peak inspiratory pressure (PIP) derived by the monitoring system attached to 


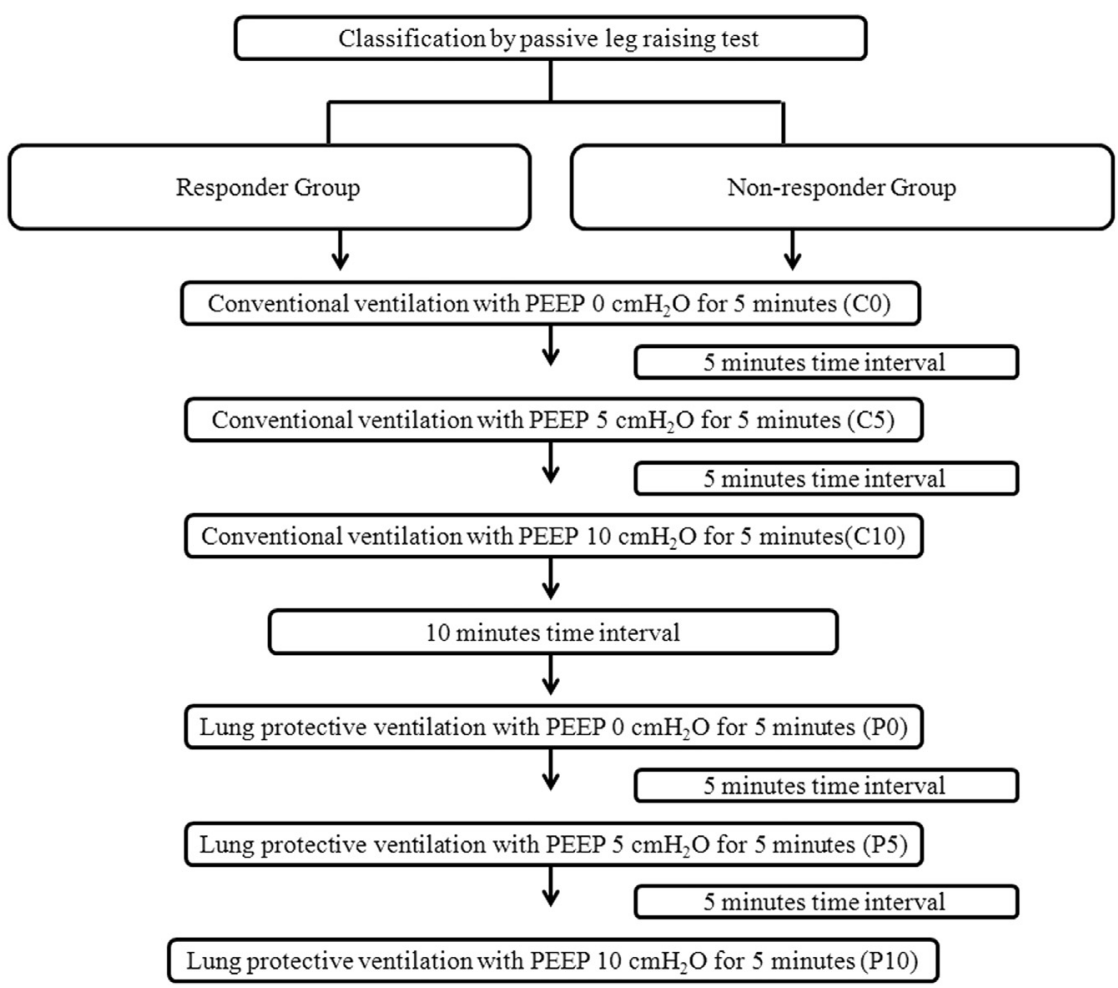

FIGURE 1. Study protocol. PEEP, Positive end-expiratory pressure.

the mechanical ventilator (Puritan Bennett 840 Ventilator System; Puritan-Bennett Corporation, Pleasanton, Calif); and (3) CO, CI, SVI, and SVV derived noninvasively using the NICOM device. Measurements for conventional ventilation began 5 minutes after return of the patient from the PLR position to the supine position, and the parameters were recorded at PEEP settings of 0 (baseline, $\mathrm{C} 0$ ), 5 (C5), and $10 \mathrm{cmH}_{2} \mathrm{O}$ (C10). The same parameters during lung protective ventilation with PEEP settings of $0(\mathrm{P} 0), 5(\mathrm{P} 5)$, and $10 \mathrm{cmH}_{2} \mathrm{O}(\mathrm{P} 10)$ were measured at 10 minutes after the first measurements. There was the interval of 10 minutes for the change of the ventilator mode, and the ventilation setting was equally set to $\mathrm{C} 0$. The parameters were recorded in the last 5 minutes after maintenance of each ventilator setting. Then, the time interval of 5 minutes was applied for each measuring point, with the mechanical ventilation settings set the same as at $\mathrm{C} 0$ or P0 (Figure 1). During the measurements, there was no change in infusion rates of inotropes, vasopressors, or fluid administration. After the evaluations of different ventilator settings, responder or nonresponder status was confirmed by $250 \mathrm{~mL}$ colloid (Voluven; Fresenius Kabi, Bad Homberg, Germany) administration at the baseline mechanical ventilation setting; if the CO measured by NICOM was increased more than $7 \%$ from the values before fluid administration, the patient was confirmed to be in group R. ${ }^{9,12}$

\section{Statistics}

The primary outcome variables were the SVV values between the 2 groups. Before our study, a pilot study with the same study protocol was performed including 10 patients who were not included in the final analysis and confirmed as nonresponders. The mean SVV values at each measured point were $10.9 \% \pm 2.6 \%(\mathrm{C} 0), 11.0 \% \pm 2.8 \%(\mathrm{C} 5), 11.3 \% \pm 2.9 \%$ (C10), $10.9 \% \pm 2.6 \%(\mathrm{P} 0), 11.4 \% \pm 2.4 \%(\mathrm{P} 5)$, and $11.1 \% \pm 2.5 \%$ (P10), calculated from the pilot study with 10 patients. For the SVV values at each measured point, a minimum of $20 \%$ difference between the 2 groups was considered to be clinically significant. Sample sizes as the following were calculated as appropriate in achieving a power of 0.8 and an alpha value of $0.05: 24$ for $\mathrm{C} 0,27$ for $\mathrm{C} 5,27$ for $\mathrm{C} 10,24$ for P0, 19 for P5, and 25 for P10. Independent 2-sample $t$ test or Mann-Whitney rank-sum test was used to compare continuous variables between groups $\mathrm{R}$ and $\mathrm{N}$ and between different ventilator settings in each group. One-way repeated-measures analysis of variance or Friedman repeated-measures analysis of variance on ranks was used for analysis of variables within each group. Categoric variables were analyzed using the chi-square test. The area under the curve (AUC) with receiver operating characteristic (ROC) curve analyses were performed in the entire population to evaluate the capacity of all hemodynamic parameters to predict the fluid responsiveness and obtain the cutoff values between groups $\mathrm{R}$ and $\mathrm{N}$. Data are expressed as numbers of patients and mean \pm standard deviations or in median (25th to 75 th percentile) values. Statistical analyses were conducted using SPSS 18.0 (SPSS Inc, Chicago, Ill).

\section{RESULTS}

During the study period, 107 aortic valve repair surgeries were performed and a total of 54 patients were eligible for inclusion in the final analysis, whereas 53 were excluded. Reasons for exclusion were other concurrent valvular surgery $(n=48)$, postoperative dysrhythmia $(n=3)$, and reoperation for postoperative bleeding in the ICU $(n=2)$.

Patient demographic profiles and preoperative diagnoses were similar between the 2 groups (Table 1), and all patients who were assigned to group $\mathrm{R}$ or $\mathrm{N}$ by the PLR test showed the same results with a $250 \mathrm{~mL}$ fluid challenge. The values of $\mathrm{CO}$ in group $\mathrm{R}$ were significantly lower than those in 
TABLE 1. Demographic data of the study group

\begin{tabular}{lccc}
\hline & Group R & Group N & P value \\
\hline Gender (male/female) & $15 / 12$ & $17 / 10$ & .58 \\
Age (y) & $49 \pm 12$ & $44 \pm 13$ & .15 \\
Height $(\mathrm{cm})$ & $167 \pm 11$ & $168 \pm 10$ & .62 \\
Weight $(\mathrm{kg})$ & $65 \pm 10$ & $70 \pm 14$ & .10 \\
Preoperative diagnosis & & & \\
$\quad$ AS & 22 & 20 & .43 \\
AR & 5 & 7 & .43 \\
\hline
\end{tabular}

$A R$, Aortic regurgitation; $A S$, aortic stenosis; group $N$, nonresponder group; group $R$, responder group.

group $\mathrm{N}$ before the PLR test and colloid administration. In group R, the values of $\mathrm{CO}$ were significantly increased after the PLR test and colloid administration (Table 2).

The PIP was the only parameter that showed significant differences in comparison between different ventilator settings in each group (group $\mathrm{R}, 15.0 \pm 4.6 \mathrm{cmH}_{2} \mathrm{O}$ at $\mathrm{C} 0$ vs $11.0 \pm 2.4 \mathrm{cmH}_{2} \mathrm{O}$ at $\mathrm{P} 0, P<.001 ; 18.8 \pm 4.5$ $\mathrm{cmH}_{2} \mathrm{O}$ at $\mathrm{C} 5$ vs $14.8 \pm 2.4 \mathrm{cmH}_{2} \mathrm{O}$ at $\mathrm{P} 5, P<.001$; $23.3 \pm 4.1 \mathrm{cmH}_{2} \mathrm{O}$ at $\mathrm{C} 10$ vs $19.8 \pm 2.1 \mathrm{cmH}_{2} \mathrm{O}$ at $\mathrm{P} 10$, $P<.001$; group $\mathrm{N}, 15.5 \pm 3.0 \mathrm{cmH}_{2} \mathrm{O}$ at $\mathrm{C} 0$ vs $11.7 \pm 3.0 \mathrm{cmH}_{2} \mathrm{O}$ at $\mathrm{P} 0, P<.001 ; 19.1 \pm 2.8 \mathrm{cmH}_{2} \mathrm{O}$ at $\mathrm{C} 5$ vs $15.7 \pm 2.3 \mathrm{cmH}_{2} \mathrm{O}$ at $\mathrm{P} 5, P<.001 ; 23.9 \pm 2.6$ $\mathrm{cmH}_{2} \mathrm{O}$ at $\mathrm{C} 10$ vs $20.3 \pm 2.0 \mathrm{cmH}_{2} \mathrm{O}$ at $\left.\mathrm{P} 10, P<.001\right)$.

There were no significant inter- or intra-group differences in MAP and heart rate during conventional ventilation (Table 3). CVP, pulmonary arterial pressure, PCWP, and PIP were increased as the PEEP level increased in both groups. CO, CI, and SVI were significantly lower in group $\mathrm{R}$ than in group $\mathrm{N}$, but they did not show intra-group differences in either group. The SVV in group $\mathrm{R}$ had an increasing trend as the PEEP level increased and was significantly higher in group $\mathrm{R}$ than in group $\mathrm{N}$ at each PEEP level $(15.8 \% \pm 3.2 \%$ in group $\mathrm{R}$ vs $10.5 \% \pm 3.3 \%$ in group $\mathrm{N}$ at $\mathrm{C} 0, P<.001 ; 17.0 \% \pm$ $2.9 \%$ in group $\mathrm{R}$ vs $10.8 \% \pm 3.5 \%$ in group $\mathrm{N}$ at $\mathrm{C} 5$, $P<.001 ; 18.0 \% \pm 2.2 \%$ in group $\mathrm{R}$ vs $11.1 \% \pm 3.4 \%$ in group $\mathrm{N}$ at $\mathrm{C} 10, P<.001)$.

In lung protective ventilation (Table 4), all parameters showed the same patterns as those in conventional ventilation. SVV also had the same patterns as conventional ventilation $(15.6 \% \pm 3.3 \%$ in group $\mathrm{R}$ vs $10.8 \% \pm 3.1 \%$ in group $\mathrm{N}$ at $\mathrm{P} 0, P<.001 ; 16.7 \% \pm 2.9 \%$ in group $\mathrm{R}$ vs $11.3 \%$ $\pm 3.2 \%$ in group $\mathrm{N}$ at $\mathrm{P} 5, P<.001 ; 17.5 \% \pm 2.6 \%$ in group $\mathrm{R}$ vs $11.1 \% \pm 3.4 \%$ in group $\mathrm{N}$ at $\mathrm{P} 10, P<.001)$.

TABLE 2. Comparison of cardiac output before and after passive leg raising test and fluid administration between the 2 groups

\begin{tabular}{lllll}
\hline & Before PLR & After PLR & Before fluid & After fluid \\
\hline Group R & $3.4(2.7-4.0)^{*}$ & $3.7(3.1-4.4) \dagger$ & $3.5(2.7-3.9)^{*}$ & $3.9(3.2-4.3) \ddagger$ \\
Group N & $4.0(3.5-4.8)$ & $4.1(3.3-4.7)$ & $4.2(3.6-4.9)$ & $4.3(3.6-5.0)$ \\
\hline
\end{tabular}

Group $N$, Nonresponder group; group $R$, responder group; $P L R$, passive leg raising. ${ }^{*} P<.05$ versus group $\mathrm{N} . \dagger P<.05$ versus before PLR test. $\ddagger P<.05$ versus before fluid administration.
The AUCs at each different PEEP in both ventilation modes were not significant for all hemodynamic parameters except SVV. The AUCs for SVV at each different PEEP in conventional ventilation were 0.899 at $\mathrm{C} 0,0.942$ at $\mathrm{C} 5$, and 0.985 at C10 (Figure 2, A). The AUCs for SVV at each different PEEP in lung protective ventilation were 0.901 at $\mathrm{P} 0,0.932$ at $\mathrm{P} 5$, and 0.947 at P10 (Figure 2, B). The optimal threshold values of SVV given by ROC curve analysis were $13.5 \%$ (sensitivity $84.6 \%$, specificity $88 \%$ ) at $\mathrm{C} 0,13.5 \%$ (sensitivity $92.3 \%$, specificity $84 \%$ ) at C5, $14.5 \%$ (sensitivity $92.3 \%$, specificity $92 \%$ ) at $\mathrm{C} 10$, $13.5 \%$ (sensitivity $88.5 \%$, specificity $88 \%$ ) at $\mathrm{P} 0,13.5 \%$ (sensitivity $92.3 \%$, specificity $84 \%$ ) at P5, and $14.5 \%$ (sensitivity $92.3 \%$, specificity $92 \%$ ) at P10 (Table 5).

\section{DISCUSSION}

In the present study, the different ventilator settings did not change the hemodynamic parameters. The SVV values were increased as PEEP levels increased regardless of conventional or lung protective ventilation in group $\mathrm{R}$, but these changes were not shown in group $\mathrm{N}$. The cutoff values for SVV in distinguishing responders and nonresponders were $13.5 \%$ at $\mathrm{C} 0,13.5 \%$ at $\mathrm{C} 5$, and $14.5 \%$ at $\mathrm{C} 10$; and $13.5 \%$ at $\mathrm{P} 0,13.5 \%$ at $\mathrm{P} 5$, and $14.5 \%$ at $\mathrm{P} 10$, respectively, and the optimal threshold values for fluid responsiveness at a PEEP of $10 \mathrm{cmH}_{2} \mathrm{O}$ in both ventilation modes were different and higher than those for a PEEP of 0 and $5 \mathrm{cmH}_{2} \mathrm{O}$.

We hypothesized that conventional ventilation may have a greater impact on the hemodynamic status of patients because of the greater TV and PIP compared with lung protective ventilation that may decrease SVI, CO, and CI and increase SVV. However, there were no significant differences in any parameter measured except PIP. We compared $6 \mathrm{~mL} \cdot \mathrm{kg}^{-1}$ of TV (lung protective ventilation) with $10 \mathrm{~mL} \cdot \mathrm{kg}^{-1}$ of TV (conventional ventilation). The PIPs in conventional ventilation with TV of $10 \mathrm{~mL} \cdot \mathrm{kg}^{-1}$ and $10 \mathrm{cmH}_{2} \mathrm{O}$ PEEP were relatively low $(23.3 \pm 4.1$ $\mathrm{cmH}_{2} \mathrm{O}$ in group $\mathrm{R}$ and $23.9 \pm 2.6 \mathrm{cmH}_{2} \mathrm{O}$ in group $\mathrm{N}$ ). The effects of PIP or inspiratory plateau pressure less than $25 \mathrm{cmH}_{2} \mathrm{O}$ were not associated with significant changes of hemodynamic parameters, such as CO, CI, and MAP, but the effect of PIP or inspiratory plateau pressures greater than $25 \mathrm{cmH}_{2} \mathrm{O}$ showed significantly decreased hemodynamic values in human and animal studies. ${ }^{13,14}$ Therefore, the limited PIP in the present study may be associated with the lack of differences in the hemodynamic measurements, although the PIP showed significant differences in different ventilator settings. If a greater TV $\left(12 \mathrm{~mL} \cdot \mathrm{kg}^{-1}\right)$ with a PIP greater than $25 \mathrm{cmH}_{2} \mathrm{O}$ in conventional ventilation had been applied, the results may have shown otherwise. In addition, we used volume-controlled ventilation in our study, and if other ventilation modes, such as pressure-controlled ventilation, 
TABLE 3. Hemodynamic parameters between responders and nonresponders during conventional ventilation

\begin{tabular}{|c|c|c|c|c|c|c|}
\hline & \multicolumn{3}{|c|}{ Group R } & \multicolumn{3}{|c|}{ Group N } \\
\hline & $\mathrm{CO}$ & C5 & $\mathrm{C10}$ & $\mathrm{CO}$ & C5 & C10 \\
\hline MAP & $86.6 \pm 10.5$ & $87.1 \pm 11.2$ & $85.7 \pm 10.9$ & $86.8 \pm 11.1$ & $86.8 \pm 12.7$ & $85.3 \pm 12.6$ \\
\hline $\mathrm{HR}$ & $82.1 \pm 11.9$ & $81.9 \pm 11.7$ & $82.0 \pm 12.2$ & $83.3 \pm 12.2$ & $83.1 \pm 12.3$ & $83.5 \pm 12.7$ \\
\hline CVP & $8.8 \pm 2.5$ & $9.8 \pm 2.7^{*}$ & $11.2 \pm 2.6^{*}, \dagger$ & $8.8 \pm 2.8$ & $9.5 \pm 2.8^{*}$ & $10.6 \pm 2.7^{*}, \dagger$ \\
\hline PAP & $19.8 \pm 3.9$ & $21.0 \pm 3.7^{*}$ & $21.9 \pm 3.8^{*}, \dagger$ & $19.4 \pm 4.8$ & $20.2 \pm 4.7^{*}$ & $21.4 \pm 4.0^{*}, \dagger$ \\
\hline PCWP & $14.2 \pm 3.5$ & $15.2 \pm 3.0$ & $17.2 \pm 3.7^{*}, \dagger$ & $14.5 \pm 4.2$ & $15.3 \pm 4.6$ & $16.8 \pm 4.0^{*}, \dagger$ \\
\hline PIP & $15.0 \pm 4.6$ & $18.8 \pm 4.5^{*}$ & $23.3 \pm 4.1^{*}, \dagger$ & $15.5 \pm 3.0$ & $19.1 \pm 2.8^{*}$ & $23.9 \pm 2.6^{*}, \dagger$ \\
\hline $\mathrm{CO}$ & $3.5(2.7-4.0) \dagger$ & $3.5(2.8-4.2) \dagger$ & $3.4(2.9-4.0) \ddagger$ & $4.4(3.4-5.1)$ & $4.3(3.5-5.4)$ & $4.5(3.4-5.9)$ \\
\hline $\mathrm{CI}$ & $1.9(1.7-2.4) \ddagger$ & $1.9(1.7-2.5) \ddagger$ & $2.0(1.6-2.3) \ddagger$ & $2.4(2.1-2.7)$ & $2.4(2.1-2.6)$ & $2.5(2.1-2.8)$ \\
\hline SVI & $23.0(21.0-29.0) \ddagger$ & $24.0(21.0-28.0) \ddagger$ & $24.0(22.0-28.0) \ddagger$ & $29.0(26.0-36.0)$ & $28.5(25.0-34.0)$ & $31.0(26.0-34.0)$ \\
\hline SVV & $15.8 \pm 3.2 \ddagger$ & $17.0 \pm 2.9^{*}, \ddagger$ & $18.0 \pm 2.2^{*}, \dagger, \ddagger$ & $10.5 \pm 3.3$ & $10.8 \pm 3.5$ & $11.1 \pm 3.4$ \\
\hline
\end{tabular}

$C I$, Cardiac index; $C O$, cardiac output; $C O$, conventional ventilation with $\mathrm{PEEP} 0 \mathrm{cmH}_{2} \mathrm{O} ; C 5$, conventional ventilation with $\mathrm{PEEP} 5 \mathrm{cmH} \mathrm{O}_{2}$; $C 10$, conventional ventilation with PEEP $10 \mathrm{cmH}_{2} \mathrm{O} ; C V P$, central venous pressure; group $N$, nonresponder group; group $R$, responder group; HR, heart rate; MAP, mean arterial pressure; $P A P$, pulmonary arterial pressure; $P C W P$, pulmonary capillary wedge pressure; $P I P$, peak inspiratory pressure; $S V I$, stroke volume index; $S V V$, stroke volume variation. $* P<.05$ versus $C 0$. $\dagger P<.05$ versus

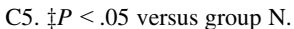

which shows lower PIP at the same TV, had been used, the results may have been different.

In postoperative mechanical ventilation management for patients undergoing cardiac surgery, PEEP is usually applied because it can improve oxygen delivery, lung compliance, and gas exchange function, and prevent atelectasis. ${ }^{15,16}$ However, PEEP can alter cardiac filling and $\mathrm{CO}$, especially postoperatively, and lead to misinterpretation of the indicators for fluid management.

The physiologic effects of mechanical ventilation influence SV of the right and left sides of the heart. In the right side of the heart, the SV of the right ventricle (RV) and the pulmonary arterial blood flow are decreased during the inspiratory phase as a result of the decreased RV preload and increased RV afterload. At same time, the left ventricle (LV) preload increases and the LV afterload decreases, resulting in increased SV of the LV. However, because the $\mathrm{SV}$ of the RV is the LV preload, the inspiratory decrease of the RV output will cause a decrease in LV filling and output after a few heartbeats. ${ }^{17-19}$ By this mechanism, the main factor affecting the absolute value of SVV may be the degree of pleural and intrathoracic pressure. Greater pleural and intrathoracic pressures may induce a greater influence on the SV in both the RV and LV, and produce a greater SVV. The results of the current study show that the optimal threshold value needed to distinguish responders from nonresponders at a PEEP of $10 \mathrm{cmH}_{2} \mathrm{O}$ was higher than the values determined at a PEEP of 0 and $5 \mathrm{cmH}_{2} \mathrm{O}$, which supports that this may be associated with greater pleural and intrathoracic pressures during a PEEP of $10 \mathrm{cmH}_{2} \mathrm{O}$. Therefore, it should be considered that the optimal threshold value of SVV may be different at different PEEP levels when SVV is used to predict fluid responsiveness.

Previous studies and several review articles about the influence of TV on the parameters in predicting fluid responsiveness have reported that the accuracy and predictability of these parameters may be increased when the TV is greater than 7 to $8 \mathrm{~mL}$ per ideal body weight. ${ }^{20-23}$ However, in the present study, even when low TV $\left(6 \mathrm{~mL} \cdot \mathrm{kg}^{-1}\right)$ of lung

TABLE 4. Hemodynamic parameters between responders and nonresponders during lung protective ventilation

\begin{tabular}{|c|c|c|c|c|c|c|}
\hline & \multicolumn{3}{|c|}{ Group R } & \multicolumn{3}{|c|}{ Group N } \\
\hline & P0 & P5 & P10 & Po & P5 & P10 \\
\hline MAP & $86.1 \pm 10.6$ & $86.8 \pm 11.6$ & $85.7 \pm 11.9$ & $85.6 \pm 11.3$ & $85.7 \pm 12.0$ & $84.4 \pm 11.6$ \\
\hline HR & $83.9 \pm 13.4$ & $83.3 \pm 13.3$ & $83.0 \pm 13.3$ & $82.2 \pm 11.4$ & $82.0 \pm 11.9$ & $81.2 \pm 11.7$ \\
\hline CVP & $8.4 \pm 2.8$ & $9.1 \pm 2.6^{*}$ & $10.4 \pm 2.5^{*}, \dagger$ & $8.7 \pm 2.5$ & $9.7 \pm 2.6^{*}$ & $10.9 \pm 2.8^{*}, \dagger$ \\
\hline PAP & $19.5 \pm 3.7$ & $20.7 \pm 3.6^{*}$ & $22.1 \pm 3.7^{*}, \dagger$ & $19.2 \pm 4.7$ & $20.1 \pm 4.5$ & $21.3 \pm 4.2^{*}, \dagger$ \\
\hline PCWP & $13.9 \pm 3.1$ & $15.4 \pm 3.0^{*}$ & $16.8 \pm 3.2^{*}, \dagger$ & $13.9 \pm 4.4$ & $15.2 \pm 4.0$ & $16.5 \pm 3.8^{*}, \dagger$ \\
\hline PIP & $11.0 \pm 2.4$ & $14.8 \pm 2.4^{*}$ & $19.8 \pm 2.1^{*}, \dagger$ & $11.7 \pm 3.0$ & $15.7 \pm 2.3^{*}$ & $20.3 \pm 2.0^{*}, \dagger$ \\
\hline $\mathrm{CO}$ & $3.5(2.8-4.0) \ddagger$ & $3.5(2.7-4.0) \ddagger$ & $3.5(2.7-3.9) \ddagger$ & $4.3(3.4-5.4)$ & $4.5(3.4-5.2)$ & $4.1(3.6-5.5)$ \\
\hline $\mathrm{CI}$ & $1.9(1.7-2.4) \ddagger$ & $2.0(1.7-2.3) \ddagger$ & $1.9(1.6-2.3) \ddagger$ & $2.4(2.1-2.7)$ & $2.4(2.1-2.8)$ & $2.4(2.2-2.7)$ \\
\hline SVI & $25.0(21.0-27.0) \ddagger$ & $24.0(22.0-28.0) \ddagger$ & $24.0(21.0-27.0) \ddagger$ & $29.0(26.0-35.0)$ & $29.0(26.0-34.0)$ & $29.5(26.0-34.0)$ \\
\hline SVV & $15.6 \pm 3.3 \ddagger$ & $16.7 \pm 2.9^{*}, \ddagger$ & $17.5 \pm 2.6^{*}, \dagger, \ddagger$ & $10.8 \pm 3.1$ & $11.3 \pm 3.2$ & $11.1 \pm 3.4$ \\
\hline
\end{tabular}

$C I$, Cardiac index; $C O$, cardiac output; $C V P$, central venous pressure; group $N$, nonresponder group; group $R$, responder group; $H R$, heart rate; $M A P$, mean arterial blood pressure; $P O$, lung protective ventilation with PEEP $0 \mathrm{cmH}_{2} \mathrm{O} ; P 5$, lung protective ventilation with PEEP $5 \mathrm{cmH}_{2} \mathrm{O} ; P 10$, lung protective ventilation with PEEP $10 \mathrm{cmH}_{2} \mathrm{O}$; PAP, mean pulmonary arterial blood pressure; $P C W P$, pulmonary capillary wedge pressure; $P I P$, peak inspiratory pressure; $S V I$, stroke volume index; $S V V$, stroke volume variation. $* P<.05$ versus $\mathrm{P} 0 . \dagger P<.05$ versus $\mathrm{P} 5 . \ddagger P<.05$ versus group $\mathrm{N}$. 

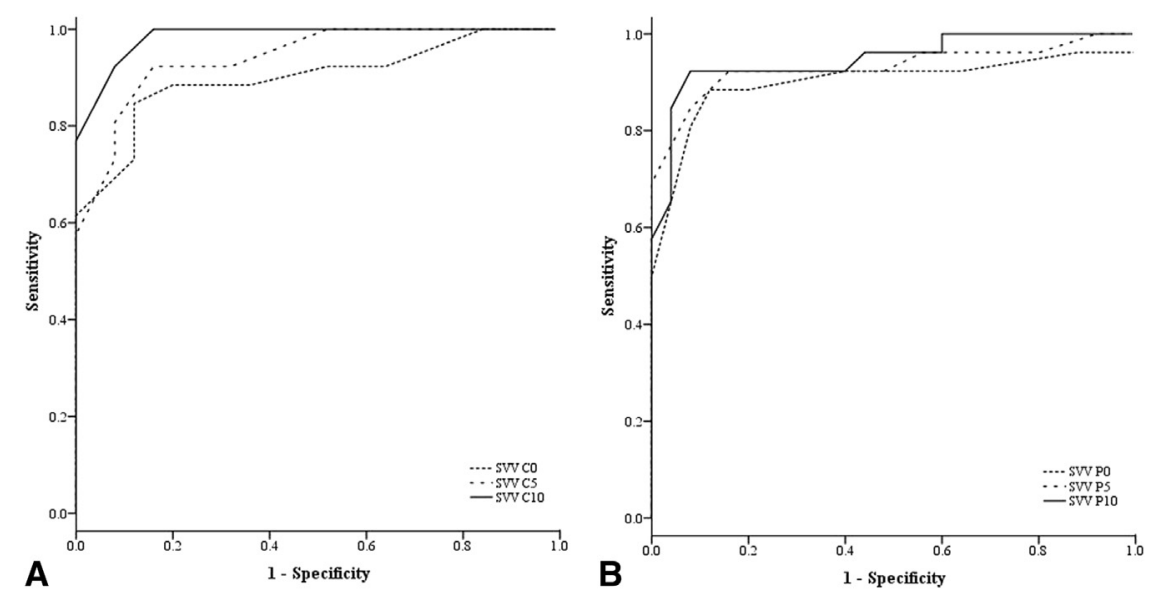

FIGURE 2. ROC curve analysis. A, Conventional ventilation. B, Lung protective ventilation. $C O$, Conventional ventilation with $\mathrm{PEEP} 0 \mathrm{cmH}_{2} \mathrm{O}$; $C 5$, conventional ventilation with PEEP $5 \mathrm{cmH}_{2} \mathrm{O} ; C 10$, conventional ventilation with $\mathrm{PEEP} 10 \mathrm{cmH}_{2} \mathrm{O}$; $P 0$, lung protective ventilation with PEEP $0 \mathrm{cmH}_{2} \mathrm{O} ; P 5$, lung protective ventilation with PEEP $5 \mathrm{cmH}_{2} \mathrm{O} ; P 10$, lung protective ventilation with PEEP $10 \mathrm{cmH}_{2} \mathrm{O} ; S V V$, stroke volume variation.

protective ventilation was applied, SVV was able to predict response or nonresponse to fluid challenge, and AUC by ROC analysis at each PEEP level showed a value comparable to that in conventional ventilation (TV 10 $\mathrm{mL} \cdot \mathrm{kg}^{-1}$ ). These results corresponded well with those of a previous study about the usefulness of measurements to predict fluid responsiveness at low TV with PEEP. ${ }^{24}$

Other methods to measure $\mathrm{CO}$ and $\mathrm{CI}$ for hemodynamic monitoring and SVV for fluid responsiveness include pulse contour analyses with patient demographic and physical characteristics (FloTrac system, Edwards Lifesciences, Irvine, Calif), which is a relatively less-invasive monitoring technique that has been used widely for monitoring $\mathrm{CO}, \mathrm{CI}$, and SVV. However, the values of arterial blood pressure and the wave-form derived from peripheral arteries might be inaccurate compared with the actual arterial blood pressure and wave-forms because the compliance and elasticity of peripheral arteries, mainly the radial artery, can be changed after weaning from cardiopulmonary bypass. ${ }^{25}$ Another recent review $^{26}$ indicated that the FloTrac system may be unreliable for estimating $\mathrm{CO}$ and $\mathrm{CI}$ compared with the

TABLE 5. Area under the curve with receiver operating characteristic curve analysis

\begin{tabular}{lcccccc}
\hline & & & $\begin{array}{c}\boldsymbol{P} \\
\text { value }\end{array}$ & $\begin{array}{c}\text { Cutoff } \\
(\%)\end{array}$ & $\begin{array}{c}\text { Sensitivity } \\
(\%)\end{array}$ & $\begin{array}{c}\text { Specificity } \\
(\%)\end{array}$ \\
\hline A0 & 0.899 & $0.810-0.988$ & $<.001$ & 13.5 & 84.6 & 88.0 \\
C5 & 0.942 & $0.881-1.000$ & $<.001$ & 13.5 & 92.3 & 84.0 \\
C10 & 0.985 & $0.891-1.000$ & $<.001$ & 14.5 & 92.3 & 92.0 \\
P0 & 0.901 & $0.803-0.998$ & $<.001$ & 13.5 & 88.5 & 88.0 \\
P5 & 0.932 & $0.839-1.000$ & $<.001$ & 13.5 & 92.3 & 84.0 \\
P10 & 0.947 & $0.865-1.000$ & $<.001$ & 14.5 & 92.3 & 92.0 \\
\hline
\end{tabular}

$P$ value: comparison with AUC $=0.5$. AUC, Area under the curve; $C I$, confidence interval; $\mathrm{CO}$, conventional ventilation with PEEP $0 \mathrm{cmH}_{2} \mathrm{O} ; C 5$, conventional ventilation with PEEP $5 \mathrm{cmH}_{2} \mathrm{O} ; \mathrm{ClO}$, conventional ventilation with PEEP 10 $\mathrm{cmH}_{2} \mathrm{O} ; P 0$, lung protective ventilation with $\mathrm{PEEP} 0 \mathrm{cmH}_{2} \mathrm{O} ; P 5$, lung protective ventilation with PEEP $5 \mathrm{cmH}_{2} \mathrm{O} ; \mathrm{PlO}$, lung protective ventilation with PEEP $10 \mathrm{cmH}_{2} \mathrm{O}$. thermodilution technique, which is considered the gold standard. This result may have been associated with inadequate measurement of SV through the pulse contour analysis. Although measurements of $\mathrm{CO}$ and $\mathrm{CI}$ using the bioreactance technique with a NICOM device is not the gold standard, NICOM does provide an alternative method for noninvasive monitoring and easy to perform measurements, and several reports have shown acceptable accuracy and usefulness of the bioreactance technique for hemodynamic status monitoring in various clinical situations. ${ }^{7,26,27}$ Therefore, we used the bioreactance technique with a NICOM device to measure CO, CI, and SVV in the present study. In addition, the patients in group $\mathrm{R}$ were confirmed through an increase of $\mathrm{CO}$ measured by a NICOM device, and other hemodynamic parameters (eg, MAP, CVP, and PCWP) had not been measured. The parameters obtained from NICOM were the mean values calculated from continuous measurements for 1 minute, but the static parameters reflect patient status only at the specific measuring point. Therefore, the $\mathrm{CO}$ measured by NICOM may be useful in more accurate monitoring of a patient's actual hemodynamic status.

\section{Study Limitations}

First, the study was observational rather than a randomized controlled trial, which means that various biases exist despite the effort to minimize them. Second, the patients with respiratory disease were excluded in the present study, although acute lung injury after cardiac surgery with cardiopulmonary bypass was possible. If the study was performed in the patients with respiratory disease, such as acute respiratory distress syndrome or chronic obstructive pulmonary disease, the different results would be due to heart-lung interactions. Third, among the patients included in this study, some had chest wall edema 
or pleural effusion, conditions that may have had an effect on our results. However, the hemodynamic status of patients was stable, so we concluded that chest wall edema or pleural effusion had a limited effect on our results.

\section{CONCLUSIONS}

The threshold values of SVV in distinguishing the fluid challenge responsiveness may be different and increase in patients receiving mechanical ventilation with a PEEP of 10 $\mathrm{cmH}_{2} \mathrm{O}$. Therefore, careful interpretation is required to prevent volume overload in this mechanical ventilation setting.

\section{References}

1. Johnson MR. Low systemic vascular resistance after cardiopulmonary bypass: are we any closer to understanding the enigma? Crit Care Med. 1999;27:1048-50.

2. Michard F, Teboul JL. Predicting fluid responsiveness in ICU patients: a critical analysis of the evidence. Chest. 2002;121:2000-8.

3. Reuter DA, Felbinger TW, Schmidt C, Kilger E, Goedje O, Lamm P, et al. Stroke volume variations for assessment of cardiac responsiveness to volume loading in mechanically ventilated patients after cardiac surgery. Intensive Care Med. 2002; 28:392-8.

4. Mesquida J, Kim HK, Pinsky MR. Effects of tidal volume, intrathoracic pressure, and cardiac contractility on variations in pulse pressure, stroke volume, and intrathoracic blood volume. Intensive Care Med. 2011;37:1672-9.

5. De Backer D, Taccone FS, Holsten R, Ibrahimi F, Vincent JL. Influence of respiratory rate on stroke volume variation in mechanically ventilated patients. Anesthesiology. 2009;110:1092-7.

6. Reuter DA, Bayerlein J, Goepfert MS, Weis FC, Kilger E, Lamm P, et al. Influence of tidal volume on left ventricular stroke volume variation measured by pulse contour analysis in mechanically ventilated patients. Intensive Care Med. 2003;29:476-80.

7. Keren H, Burkhoff D, Squara P. Evaluation of a noninvasive continuous cardiac output monitoring system based on thoracic bioreactance. Am J Physiol Heart Circ Physiol. 2007;293:H583-9.

8. Marqué S, Cariou A, Chiche JD, Squara P. Comparison between Flotrac-Vigileo and Bioreactance, a totally noninvasive method for cardiac output monitoring. Crit Care. 2009; 13:R73.

9. Lafanechère A, Pène F, Goulenok C, Delahaye A, Mallet V, Choukroun G, et al. Changes in aortic blood flow induced by passive leg raising predict fluid responsiveness in critically ill patients. Crit Care. 2006;10:R132.

10. Benomar B, Ouattara A, Estagnasie P, Brusset A, Squara P. Fluid responsiveness predicted by noninvasive bioreactance-based passive leg raise test. Intensive Care Med. 2010;36:1875-81.

11. Brower RG, Lanken PN, MacIntyre N, Matthay MA, Morris A, Ancukiewicz M, et al. Higher versus lower positive end-expiratory pressures in patients with the acute respiratory distress syndrome. $N$ Engl $\mathrm{J} \mathrm{Med}$. 2004;351:327-36.

12. Solus-Biguenet H, Fleyfel M, Tavernier B, Kipnis E, Onimus J, Robin E, et al. Non-invasive prediction of fluid responsiveness during major hepatic surgery. Br J Anaesth. 2006;97:808-16.

13. Krebs J, Pelosi P, Tsagogiorgas C, Alb M, Luecke T. Effects of positive end-expiratory pressure on respiratory function and hemodynamics in patients with acute respiratory failure with and without intra-abdominal hypertension: a pilot study. Crit Care. 2009;13:R160.

14. Regli A, Hockings LE, Musk GC, Roberts B, Noffsinger B, Singh B, et al Commonly applied positive end-expiratory pressures do not prevent functiona residual capacity decline in the setting of intra-abdominal hypertension: a pig model. Crit Care. 2010;14:R128.

15. Dongelmans DA, Hemmes SN, Kudoga AC, Veelo DP, Binnerkade JM Schultz MJ. Positive end-expiratory pressure following coronary artery bypass grafting. Minerva Anestesiol. 2012;78:790-800.

16. Marino PL. Patterns of assisted ventilation. In: Marino PL, ed. The ICU Book. 2nd ed. Baltimore, MD: Williams \& Wilkins; 1997:434-48.

17. Michard F. Changes in arterial pressure during mechanical ventilation Anesthesiology. 2005;103:419-28; quiz 449-5.

18. Michard F, Teboul JL. Using heart-lung interactions to assess fluid responsiveness during mechanical ventilation. Crit Care. 2000;4:282-9.

19. Bendjelid K, Romand JA. Fluid responsiveness in mechanically ventilated patients: a review of indices used in intensive care. Intensive Care Med. 2003 29:352-60.

20. De Backer D, Heenen S, Piagnerelli M, Koch M, Vincent JL. Pulse pressure variations to predict fluid responsiveness: influence of tidal volume. Intensive Care Med. 2005;31:517-23.

21. Charron C, Fessenmeyer C, Cosson C, Mazoit JX, Hebert JL, Benhamou D, et al The influence of tidal volume on the dynamic variables of fluid responsiveness in critically ill patients. Anesth Analg. 2006;102:1511-7.

22. Zhang Z, Lu B, Sheng X, Jin N. Accuracy of stroke volume variation in predicting fluid responsiveness: a systemic review and meta-analysis. J Anesth. 2011;25:904-16

23. Cannesson M, Aboy M, Hofer CK, Rehman M. Pulse pressure variation: where are we today? J Clin Monit Comput. 2011;25:45-56.

24. Huang CC, Fu JY, Hu HC, Kao KC, Chen NH, Hsieh MJ, et al. Prediction of fluid responsiveness in acute respiratory distress syndrome patients ventilated with low tidal volume and high positive end-expiratory pressure. Crit Care Med. 2008;36:2810-6.

25. Kanazawa M, Fukuyama H, Kinefuchi Y, Takiquchi Y, Suzuki T Relationship between aortic-to-radial arterial pressure gradient after cardiopulmonary bypass and changes in arterial elasticity. Anesthesiology. 2003;99: 48-53.

26. Marik PE. Noninvasive cardiac output monitors: a state-of the-art review J Cardiothorac Vasc Anesth. 2013;27:121-34.

27. Raval NY, Squara P, Cleman M, Yalamanchili K, Winklmaier M, Burkhoff D. Multicenter evaluation of noninvasive cardiac output measurement by bioreactance technique. J Clin Monit Compt. 2008;22:113-9. 